University of Wollongong

Research Online

Faculty of Engineering - Papers (Archive)

Faculty of Engineering and Information

Sciences

$1-1-2006$

\title{
Robust motion tracking control of piezoelectric actuation systems
}

Hwee Choo Liaw

Monash University

Denny Oetomo

Monash University

Bijan Shirinzadeh

Monash University

Gursel Alici

University of Wollongong, gursel@uow.edu.au

Follow this and additional works at: https://ro.uow.edu.au/engpapers

Part of the Engineering Commons

https://ro.uow.edu.au/engpapers/4215

\section{Recommended Citation}

Liaw, Hwee Choo; Oetomo, Denny; Shirinzadeh, Bijan; and Alici, Gursel: Robust motion tracking control of piezoelectric actuation systems 2006, 1414-1419.

https://ro.uow.edu.au/engpapers/4215

Research Online is the open access institutional repository for the University of Wollongong. For further information contact the UOW Library: research-pubs@uow.edu.au 


\section{Robust Motion Tracking Control of Piezoelectric Actuation Systems}

\author{
Hwee Choo Liaw, Denny Oetomo, Bijan Shirinzadeh \\ Robotics and Mechatronics Research Laboratory \\ Department of Mechanical Engineering \\ Monash University, Clayton, VIC 3800, Australia \\ \{hwee.liaw, denny.oetomo, bijan.shirinzadeh\}@eng.monash.edu.au
}

\author{
Gursel Alici \\ School of Mechanical, Materials, \\ and Mechatronic Engineering \\ University of Wollongong, NSW 2522, Australia \\ gursel@uow.edu.au
}

\begin{abstract}
This paper proposes a robust control methodology for piezoelectric actuation systems to track specified motion trajectories. This is motivated by the search for an effective control strategy to deal with the problem of nonlinear behaviour in the piezoelectric actuation systems. The basic concept associated with this approach lies in the specification of a target performance and the formulation of a robust control scheme for the system to ensure the convergence of the position tracking error to zero in the presence of parametric uncertainties and hysteresis effect inclusive of other un-modelled disturbances. Stability of the control system is proven theoretically and the robust control methodology is demonstrated to possess a promising tracking ability through the control experiments. Implementation of the control law requires only a knowledge of the estimated parameters and their corresponding bounds as well as the bound of the hysteresis effect including disturbances. Being capable of handling uncertainties and disturbances, the robust control methodology is very attractive in the field of micro/nanomanipulation in which high-precision control applications could be realised.
\end{abstract}

\section{INTRODUCTION}

Micro/nano manipulation has been identified as one of the key enabling technologies for many research frontiers such as biomedical engineering, micro manufacturing and assembly, nanotechnology, nano robotics, and micro surgery, to name a few. In achieving these high-precision tasks, piezoelectric systems have been identified as an effective means of motion actuation, due to their high stiffness, fast response, and physically unlimited resolution. In recent years, advancements in piezoelectric actuator (PEA) designs and sensing devices such as laser interferometer encoders, capacitive sensors, strain gauges, and linear variable displacement transducers, and developments of flexure-based mechanisms [1]-[3] have further boosted the importance of the micro/nano manipulation systems in the steadily growing technological areas.

One major drawback of the PEAs is the presence of highly nonlinear hysteretic behaviour between the input (applied) voltage and the output displacement. This prevents the PEA from providing the desired high-precision motion resolution and accuracy. Research has been conducted in this area to model and compensate for the hysteresis effect. Some examples include the modelling of physical hysteresis [4], a dynamic model of hysteresis for a bi-morph beam [5], a comprehensive voltage-input electromechanical model [6], a differential model of hysteresis and its identification [7], and a charge steering model that bypasses the hysteretic problem coupled with a comprehensive model of mechanical dynamics of the PEA [8].

On the other hand, appropriate control strategies can be formulated to take the non-linearities into account to achieve high-precision positioning of the PEA systems. Recent examples include a combination of a feed-forward model in feedback control with an input shaper [9], an adaptive backstepping approach [10], a PID-based control with iterative learning plus a disturbance observer [11], a model-based open-loop control [12], a nonlinear observer-based variable structure control [13], and a sliding mode control augmented with an inverse hysteresis model [14]. Most of these control strategies are formulated either for specific applications and/or implemented for point-to-point tracking of reference positions.

In this paper, a robust control methodology is proposed to track motion trajectories in the PEA systems. This is motivated by the presence of nonlinear behaviour in the PEA systems, which makes the exact parameter values of the model difficult to identify. In this control strategy, the variable structure control approach [15] is employed to deal with not only the parametric uncertainties but also the hysteresis effect in the PEA systems including other un-modelled disturbances. The basic concept in this approach lies in the specification of a target performance and the formulation of a robust control law for a PEA system to drive its position tracking error to converge to zero. The proposed robust control methodology can steer the PEA system to reach the specified target performance and drive the system to closely follow any specified desired motion trajectory in position, velocity, and acceleration. This control scheme is unique as a dynamic compensator is introduced to form a switching function such that the resulting sliding mode can be matched with the target performance.

The stability of the robust control methodology is proven theoretically and the control system is demonstrated to possess a promising tracking ability through control experiments. Implementation of the proposed control law requires only a knowledge of the estimated system parameters and their corresponding bounds as well as the bound of the hysteresis effect including disturbances. With the inherent capability to handle parametric uncertainties and accommodate disturbances, the 


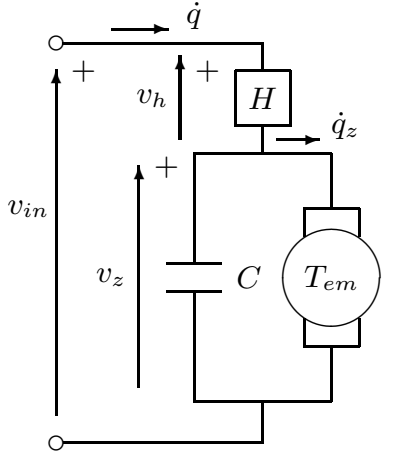

Voltage-Charge:

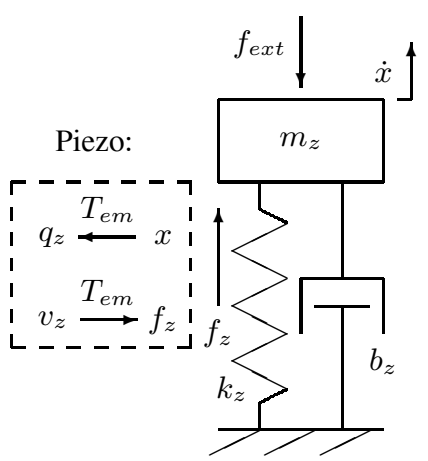

Force-Displacement:
Fig. 1. Schematic model of a piezoelectric actuator

robust control methodology is deemed as a suitable candidate for the control of the micro/nano manipulation systems.

This paper is organised as follows. The model of a piezoelectric actuator is presented in Section II, a target performance is introduced in Section III, and modelling of uncertainties is described in Section IV. A robust control methodology is formulated in Section $\mathrm{V}$ followed by an experimental study in Section VI. The results are presented and discussed in Section VII, and finally, conclusions are drawn in Section VIII.

\section{Model of Piezoelectric Actuator}

An electromechanical model of a PEA is given in [6] and [8]. This mathematical model can be divided into three stages of transformation from electrical to mechanical energy, and vice versa. The schematic model, as shown in Fig. 1, illustrates the transformation, which consists of the voltagecharge stage, piezo stage, and force-displacement stage. Note that the model in Fig. 1 is formulated for a voltage-controlled amplifier. The dynamic equation from the electrical input to the output motion stage can be described by the following set of equations:

$$
\begin{aligned}
v_{i n} & =v_{h}+v_{z}, \\
v_{h} & =H(q), \\
q & =C v_{z}+q_{z}, \\
q_{z} & =T_{e m} x, \\
f_{z} & =T_{e m} v_{z} \\
m_{z} \ddot{x}+b_{z} \dot{x}+k_{z} x & =f_{z}-f_{\text {ext }},
\end{aligned}
$$

where $v_{i n}$ represents the applied (input) voltage, $v_{h}$ is the voltage due to the hysteresis, $v_{z}$ is the voltage related to the mechanical side of the actuator, $q$ is the total charge in the ceramic, $H$ is the hysteresis effect, $C$ is the linear capacitance connected in parallel with the electromechanical transformer having a ratio of $T_{e m}, q_{z}$ is the piezo charge related to the actuator output displacement $x$, and $f_{z}$ is the transduced force from the electrical domain. The variables $m_{z}, b_{z}$, and $k_{z}$ are the mass, damping, and stiffness, respectively, of the forcedisplacement stage, and $f_{\text {ext }}$ is the force imposed by the external mechanical load. In PEA, hysteresis causes a highly nonlinear input/output relationship between the applied voltage and displacement. Goldfarb and Celanovic [6] described the hysteresis effect as a nonlinear charge-dependent phenomenon and it appears only in the electrical domain.

For control purposes, (1) and (5) are substituted into (6) to yield

$$
m_{z} \ddot{x}+b_{z} \dot{x}+k_{z} x=T_{e m}\left(v_{i n}-v_{h}\right)-f_{\text {ext }},
$$

and the PEA model is obtained by re-arranging (7),

$$
m \ddot{x}+b \dot{x}+k x+v_{h}+f_{e}=v_{i n},
$$

where $m=m_{z} / T_{e m}, b=b_{z} / T_{e m}, k=k_{z} / T_{e m}$, and $f_{e}=$ $f_{\text {ext }} / T_{\text {em }}$.

\section{TARGET PERFORMANCE}

In the control of a system, it is desired that the system follows a specified motion trajectory with a goal, which is defined as the target performance.

It is assumed that there exists an ideal model of (8), given as:

$$
m_{d} \ddot{x}+b_{d} \dot{x}+k_{d} x+v_{h}+f_{e}=v_{i n},
$$

where $m_{d}, b_{d}$, and $k_{d}$ are the desired constant values of mass, damping, and stiffness of the system, respectively, and the $v_{h}$ and $f_{e}$ are known. The system (9) is commanded to follow a desired motion trajectory $x_{d}(t)$ with the desired command:

$$
v_{d}=m_{d} \ddot{x}_{d}+b_{d} \dot{x}_{d}+k_{d} x_{d}+v_{h}+f_{e} .
$$

If the desired command in (10) is exactly the same as applied voltage in (9), i.e. $v_{d}=v_{i n}$, then the target performance can be formulated by combining these equations and is given by the error function:

$$
m_{d} \ddot{e}_{p}+b_{d} \dot{e}_{p}+k_{d} e_{p}=0,
$$

where $e_{p}(t)=x(t)-x_{d}(t)$. With suitable choice of $m_{d}, b_{d}$, and $k_{d}$, the error function (11) is stable (i.e. $e_{p} \rightarrow 0$ ) and describes the closed-loop dynamics of the system.

\section{Modelling of UnCertainties}

In practice, the exact parameters of the system (8) are difficult, if not impossible, to determine. The modelling of parametric uncertainties is therefore introduced. It is stated that the exact values of $m, b$, and $k$ in (8) may not be known, however the estimated values are available. The modelling of uncertainties can also be extended to include the nonlinear hysteresis in (8) and it is assumed that both the parametric errors and hysteresis are bounded:

$$
\begin{aligned}
\|\Delta m\| & =\|m-\hat{m}\| \leq \delta m \\
\|\Delta b\| & =\|b-\hat{b}\| \leq \delta b \\
\|\Delta k\| & =\|k-\hat{k}\| \leq \delta k \\
\left\|v_{h}\right\| & \leq \delta v_{h},
\end{aligned}
$$

where $\Delta \bullet$ represents the modelling error of $\bullet$ and $\hat{\bullet}$ represents the estimated values of $\bullet$. The symbol $\|\bullet\|$ denotes an absolute value of $\bullet$ and the positive values $\delta m, \delta b, \delta k$, and $\delta v_{h}$ denote the bounds of the variables. These bounds are assumed to be 
known. Generally, the bound $\delta v_{h}$ could be further extended to incorporate other un-modelled disturbances in the system provided that the overall bound of the uncertainties is known.

\section{Robust Control Methodology}

The motion tracking control problem in the PEA system can be formulated as a target performance reaching problem in designing a control law such that the system described by (8) achieves the target performance (11) and follows the required trajectory $x_{d}(t)$ under parametric uncertainties and hysteresis (12). In the following, a robust control methodology is formulated to solve the problem.

To achieve robust control, a switching function $\sigma$ is first specified,

$$
\sigma=\dot{e}_{p}+\xi,
$$

where $\xi$ is the state of a dynamic compensator used to shape the tracking errors. The dynamic compensator can be designed as

$$
\dot{\xi}=\alpha \xi+k_{p} e_{p}+k_{v} \dot{e}_{p},
$$

where $\alpha$ is a constant scalar, $\alpha \leq 0, k_{p}$ and $k_{v}$ are the control gains which are related to the specified target performance (11). Differentiating (13) with respect to time,

$$
\dot{\sigma}=\ddot{e}_{p}+\dot{\xi} .
$$

To examine the closed-loop dynamics of the system under the sliding mode control, the dynamic compensator (14) is substituted into (15) with the term $\xi$ in (14) eliminated by using (13),

$$
\ddot{e}_{p}+\left(k_{v}-\alpha\right) \dot{e}_{p}+k_{p} e_{p}=\dot{\sigma}-\alpha \sigma .
$$

By choosing

$$
\begin{aligned}
& k_{p}=m_{d}^{-1} k_{d}, \\
& k_{v}=m_{d}^{-1} b_{d}+\alpha,
\end{aligned}
$$

the closed-loop dynamics (16) becomes

$$
m_{d} \ddot{e}_{p}+b_{d} \dot{e}_{p}+k_{d} e_{p}=m_{d}(\dot{\sigma}-\alpha \sigma) .
$$

During sliding motion where $\dot{\sigma}=0$ and $\sigma=0$, (18) achieves the target performance (11). A control law can therefore be formulated to drive the system to reach the sliding mode.

Theorem: For the system described by (8) under parametric uncertainties and hysteresis (12), the system achieves the target performance (11) with the following robust control law

$$
v_{i n}=\hat{m} \ddot{x}_{e q}+\hat{b} \dot{x}+\hat{k} x+f_{e}-k_{s} \sigma-d \frac{\sigma}{\|\sigma\|},
$$

where

$$
\ddot{x}_{e q}=\ddot{x}_{d}-\dot{\xi},
$$

and the term $d$ is governed by

$$
d \geq \delta m\left\|\ddot{x}_{e q}\right\|+\delta b\|\dot{x}\|+\delta k\|x\|+\delta v_{h}+\epsilon .
$$

The terms $k_{s}$ and $\epsilon$ in (19) and (21), respectively, are any positive scalars.
Proof: For the system described by (8) with the control law (19), a Lyapunov function $u(\sigma)$ is proposed,

$$
u(\sigma)=\frac{1}{2} m \sigma^{2},
$$

which is continuous and non-negative. Differentiating $u(\sigma)$ with respect to time yields

$$
\dot{u}(\sigma)=m \sigma \dot{\sigma} .
$$

From (15) and (20),

$$
\dot{\sigma}=\ddot{x}-\ddot{x}_{e q},
$$

and (23) is rewritten as

$$
\begin{aligned}
\dot{u}(\sigma) & =\sigma\left(m \ddot{x}-m \ddot{x}_{e q}\right) \\
& =\sigma\left(v_{i n}-b \dot{x}-k x-v_{h}-f_{e}-m \ddot{x}_{e q}\right)
\end{aligned}
$$

where $v_{i n}$ is obtained from (8). Substituting the control law (19) to replace $v_{i n}$ and using the bounds (12),

$$
\begin{aligned}
\dot{u}(\sigma)= & -k_{s} \sigma^{2}-d\|\sigma\|+\sigma\left[-\Delta m \ddot{x}_{e q}-\Delta b \dot{x}\right. \\
& \left.-\Delta k x-v_{h}\right], \\
\leq & -k_{s} \sigma^{2}-d\|\sigma\|+\|\sigma\|\left[\left\|\Delta m \ddot{x}_{e q}\right\|+\|\Delta b \dot{x}\|\right. \\
& \left.+\|\Delta k x\|+\left\|v_{h}\right\|\right], \\
\leq & -k_{s} \sigma^{2}-d\|\sigma\|+\|\sigma\|\left[\delta m\left\|\ddot{x}_{e q}\right\|+\delta b\|\dot{x}\|\right. \\
& \left.+\delta k\|x\|+\delta v_{h}\right] .
\end{aligned}
$$

From (21),

$$
\dot{u}(\sigma) \leq-k_{s} \sigma^{2}-\epsilon\|\sigma\| .
$$

This shows that $u(\sigma) \rightarrow 0$ (which in turn implies that $\sigma \rightarrow 0$ ) as $t \rightarrow \infty$. Both the stability of the system and convergence of the tracking are guaranteed by the robust control law (19) driving the system (8) to reach the target performance (11).

In the implementation of the control law (19), the discontinuous function $\frac{\sigma}{\|\sigma\|}$ will give rise to control chattering due to imperfect switching in the computer control. This is undesirable as un-modelled high frequency dynamics might be excited. To eliminate this effect, the concept of boundary layer technique [16] is applied to smooth the control signal. In a small neighborhood of the sliding surface $(\sigma=0)$, the discontinuous function is replaced by a saturation function which is defined as

$$
\operatorname{sat}\left(\frac{\sigma}{\Delta}\right)=\left\{\begin{array}{lll}
-1 & : & \sigma<-\Delta \\
\sigma / \Delta & : & -\Delta \leq \sigma \leq \Delta, \\
+1 & : & \sigma>\Delta
\end{array}\right.
$$

where $\Delta$ is the boundary layer thickness, and the control law (19) becomes

$$
v_{i n}=\hat{m} \ddot{x}_{e q}+\hat{b} \dot{x}+\hat{k} x+f_{e}-k_{s} \sigma-d \operatorname{sat}\left(\frac{\sigma}{\Delta}\right) .
$$

With the introduction of the saturation function (28) in the control law (29), the accuracy of $\sigma$ can only be guaranteed to stay within the boundary layer. From the closed-loop dynamics 


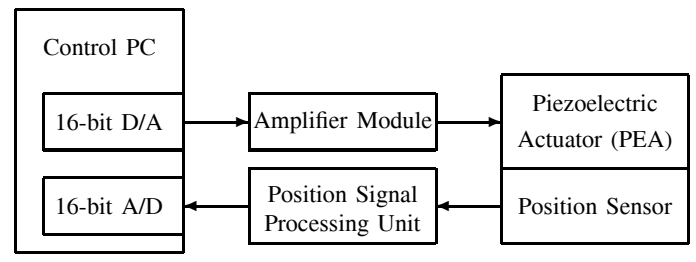

Fig. 2. Block diagram of PEA experimental system

(18) of the control law, the steady-state value $\sigma_{s s}$ of the switching function within the boundary layer is given as

$$
\sigma_{s s}=-\frac{k_{d} e_{p s s}}{m_{d} \alpha}
$$

where $e_{p s s}$ is the steady-state position error. As (30) describes the relationship between the steady-state position error and switching function, it can therefore be used to decide on the boundary layer thickness $\Delta$ in the control implementation.

The selection of a target performance for the control system is straightforward. By comparing (11) to a standard secondorder characteristic equation

$$
s^{2}+2 \zeta w_{n} s+w_{n}^{2}=0,
$$

where $s, \zeta$, and $w_{n}$ are the Laplace operator, damping ratio, and undamped natural frequency, respectively, the desired parameters are obtained as

$$
m_{d}=1, \quad b_{d}=2 \zeta w_{n}, \quad k_{d}=w_{n}^{2} .
$$

As the desired response is selected through $\zeta$ and $w_{n}$, the control gains $k_{p}$ and $k_{v}$ in (17) can therefore be calculated from (32).

\section{EXPERIMENTAL STUDY}

In the process of developing a micro/nano manipulation system using the PEAs, a single-axis PEA is set up for the experimental study of the proposed robust control methodology. The schematic of the experimental system, as shown in Fig. 2, consists of a PEA with position sensor, an amplifier module, a position signal processing unit, and a control PC installed with a digital-to-analogue (D/A) and an analog-todigital (A/D) boards. The PEA employed is a PI (Physik Instrumente) multilayer PZT stacked ceramic translator, model P-843.30, capable of expansion of up to $45 \mu \mathrm{m}$ corresponding to a range of operating voltage up to $100 \mathrm{~V}$. The PEA is preloaded $300 N$ by an internal spring and is incorporated with a high-resolution strain gauge sensor for position feedback. The amplifier module is a PI model E-505.00 with a fixed output gain of 10 providing voltage ranges from -20 to +120 $V$. The position signal processing unit is housed in a PI servo controller, model E-509-X3. The PI servo controller is disabled and only the signal processing unit is used to interface with the PEA position sensor. A standard desktop computer is used as the control PC. It is equipped with a Pentium $42.8 \mathrm{GHz}$ processor running on an operating system capable of hard realtime control. The D/A and A/D boards installed in the control $\mathrm{PC}$ are of 16-bit resolution, and they are used to generate the
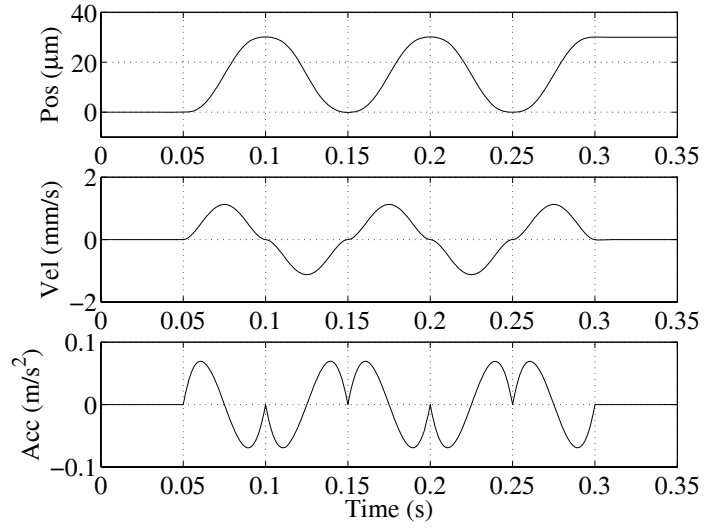

Fig. 3. Desired motion trajectory

control signal and to read the analog position, respectively. In the control experiments, the sampling frequency of the control loop is set at $2.5 \mathrm{kHz}$.

The control experiments serve not only to validate the theoretical formulation of the control algorithm but also to examine the effectiveness of the proposed scheme in a physical PEA system. In the experimental study, the closed-loop system is required to follow a desired motion trajectory, which is shown in Fig. 3 for position, velocity, and acceleration. The desired motion trajectory is formed by segments of quintic polynomials [17] for the implementation and analysis of the tracking and steady-state performances of the system.

For the PEA system described by (8), the control law (29) is implemented in the control PC as shown in Fig. 2. With the desired motion trajectory, the tracking ability of the control system can be closely examined when it is subjected to parametric uncertainties and unknown disturbance including the hysteresis effect.

To study the effect of the proposed robust control methodology, a model-based control scheme is also implemented for comparison by omitting the parametric uncertainties and hysteresis effect in the PEA system (8). Assuming that the ideal system is modelled as

$$
\hat{m} \ddot{x}+\hat{b} \dot{x}+\hat{k} x+f_{e}=v_{i n},
$$

where $\hat{m}, \hat{b}$, and $\hat{k}$ are the known parameters, the model-based control scheme can be realised as

$$
v_{\text {in }}=\hat{m} \ddot{x}^{*}+\hat{b} \dot{x}+\hat{k} x+f_{e},
$$

with

$$
\ddot{x}^{*}=\ddot{x}_{d}-m_{d}^{-1}\left(b_{d} \dot{e}_{p}+k_{d} e_{p}\right) .
$$

It must be noted that for the ideal system (33) with the modelbased control scheme (34) and (35), the close-loop dynamics can be derived and expressed as the target performance (11).

Table I summarises the parameter values of the PEA model (8) for the control experiments. From Table I, the estimated values are obtained from the open-loop tests of the PEA system. These estimated values are also chosen for the bounds of the parametric uncertainties. The bound of the hysteresis 
TABLE I

PARAMETERS FOR PEA CONTROL SYSTEM

\begin{tabular}{|l|l|l|}
\hline & Estimated Values: & Bounds: \\
\hline Mass $\left(V s^{2} / m\right):$ & $\hat{m}=2$ & $\delta m=2$ \\
\hline Damping $(V s / m):$ & $\hat{b}=3 \times 10^{3}$ & $\delta b=3 \times 10^{3}$ \\
\hline Stiffness $(V / m):$ & $\hat{k}=2.5 \times 10^{6}$ & $\delta k=2.5 \times 10^{6}$ \\
\hline Hysteresis $(V):$ & & $\delta v_{h}=20$ \\
\hline
\end{tabular}
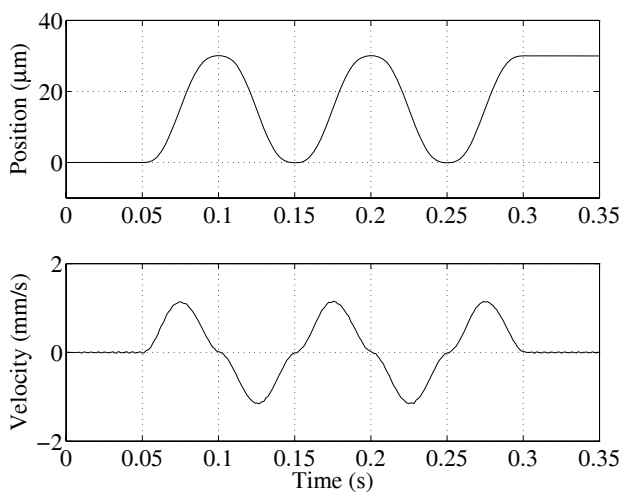

Fig. 4. Robust control: Actual position and estimated velocity

is selected differently. This value is based on the fact that hysteresis is usually in the order of $10 \%$ to $15 \%$ of the commanded motion as mentioned in the specification of the PEA system [18].

For an undamped natural frequency of $w_{n}=$ $1256.64(\mathrm{rad} / \mathrm{s})$ and a critically damped response, $\zeta=1.0$, the desired parameters in (32) are calculated as

$$
m_{d}=1, \quad b_{d}=2513, \quad \text { and } \quad k_{d}=1579,137,
$$

where the units of $m_{d}, b_{d}$, and $k_{d}$ are $\left(V s^{2} / m\right),(V s / m)$, and $(V / m)$, respectively. The constant scalar $\alpha$ in (14) is set as $\alpha=-1(1 / s)$ arbitrarily and the control gains $k_{p}$ and $k_{v}$ in (17) are calculated from (36) as

$$
k_{p}=1579,137\left(1 / s^{2}\right) \quad \text { and } \quad k_{v}=2512(1 / s) .
$$

The steady-state position error in (30) is specified as $e_{p s s} \leq$ $0.1(\mu \mathrm{m}), \sigma_{s s} \leq 0.16(\mathrm{~m} / \mathrm{s})$, the boundary layer thickness $\Delta$ in (28) is chosen as the maximum value of $\sigma_{s s}$, i.e.

$$
\Delta=0.16(\mathrm{~m} / \mathrm{s}) \text {. }
$$

The positive scalar $\epsilon$ in (21) is specified as $\epsilon=1(V)$ and $k_{s}$ of the control law (29) is set to $k_{s}=500(\mathrm{Vs} / \mathrm{m})$.

For comparison, the model-based control scheme (34) and (35) is implemented using the same estimated parameters and desired parameters as listed in Table I and (36), respectively.

\section{RESUlTS AND Discussion}

The experimental results of the proposed robust control methodology are shown in Fig. 4 to Fig. $7 . \quad$ In tracking of the desired motion trajectory as shown in Fig. 3, the PEA was commanded to travel in a range of $30 \mu \mathrm{m}$ with maximum

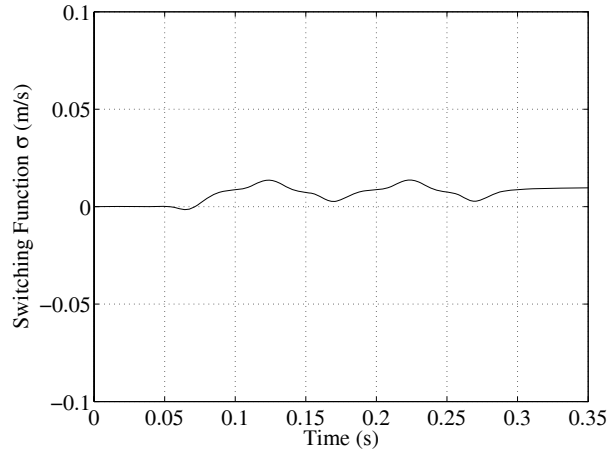

Fig. 5. Robust control: Switching function
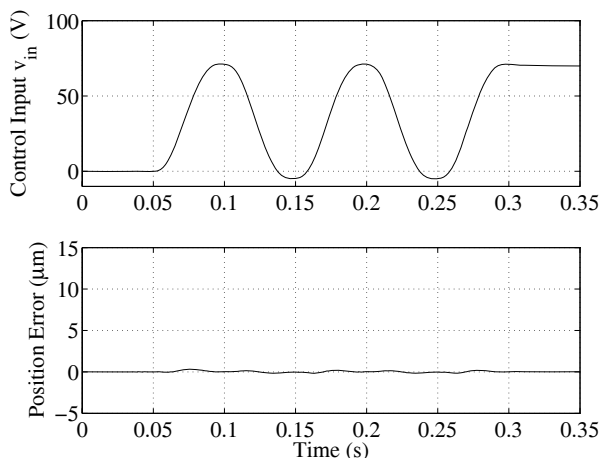

Fig. 6. Robust control: Control input and position tracking error

velocity and acceleration reaching $1.1 \mathrm{~mm} / \mathrm{s}$ and $0.07 \mathrm{~m} / \mathrm{s}^{2}$, respectively. The resulting PEA position and estimated velocity are shown in Fig. 4. Despite parametric uncertainties, hysteresis effect and external disturbances in the system, the control law (29) showed a promising tracking performance. The switching function, as shown in Fig. 5, indicates that the system operated within the boundary layer thickness given in (38), i.e. the system tracked the desired motion trajectory closely with the switching function $\sigma$ kept to a minimum. The control input and position tracking error are shown in Fig. 6. The resulting position tracking error indicates that the control law had successfully accommodated the hysteresis, without any feed-forward model, treating the hysteresis purely as an

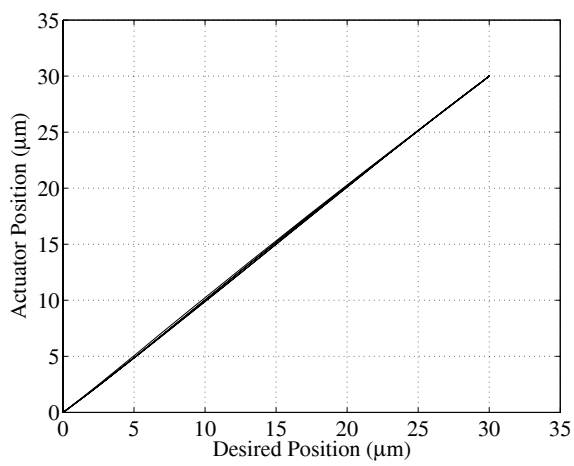

Fig. 7. Robust control: Actuator position against desired position 

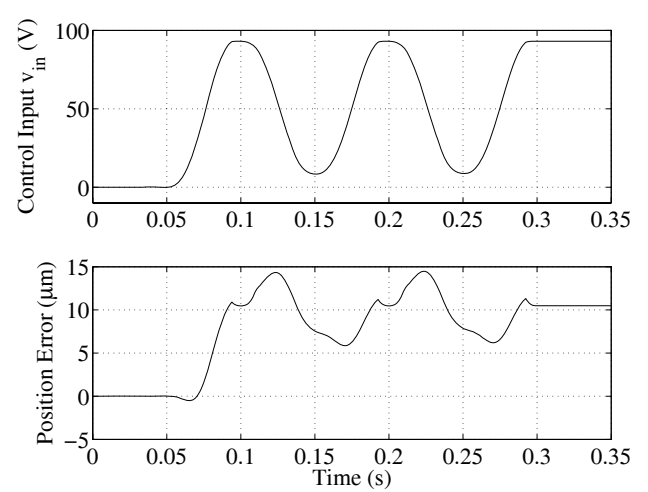

Fig. 8. Model-base control: Control input and position tracking error

external disturbance. In the experiment, the position tracking error was confined within $0.35 \mu \mathrm{m}$ during motion and less than $0.03 \mu \mathrm{m}$ at steady-state, which was almost at the noise level of the closed-loop system. Fig. 7 shows the resulting actuator position when plotted against the desired position with a minimum hysteresis effect. This shows the effectiveness of the proposed robust control methodology.

In comparison, the experimental results of the model-based control scheme (34) and (35) are shown in Fig. 8. The modelbased control resulted in a relatively large position tracking error, which was more than $14 \mu \mathrm{m}$. Without taking into account the parametric uncertainties and disturbances, the model-based control scheme proved to be comparatively less effective for the tracking of motion trajectory.

On the whole, the robust control methodology is shown to be stable, robust, and capable of following the desired motion trajectory. This is possible as the robust control methodology is able to handle parametric uncertainties and disturbances, forcing the closed-loop system to reach the specified target performance. The control implementation is appropriate and requires only the estimated parameters and the bounds of the system.

\section{CONCLUSIONS}

A robust control methodology has been proposed for the piezoelectric actuation systems to follow specified motion trajectories. The basic concept in this approach lies in the specification of a target performance and the formulation of a robust control law for a PEA system to drive its position tracking error to converge to zero.

This control scheme is unique as a dynamic compensator is introduced to form the switching function such that the resulting sliding mode can be matched with the target performance. Implementation of the control law is practical and requires only a knowledge of the estimated system parameters and their corresponding bounds as well as the bound of the hysteresis effect including external disturbances.

Stability of the robust control methodology has been proven theoretically and the control system is demonstrated to possess a promising tracking ability through the control experiments.
Being capable of handling parametric uncertainties and disturbances, the robust control methodology is very attractive in the field of micro/nano-manipulation in which high-precision PEA control applications can be realised.

\section{ACKNOWLEDGMENT}

This work is supported by an Australian Research Council LIEF grant, an Australian Research Council Discovery grant, and the Monash Research Fund.

\section{REFERENCES}

[1] S. S. Ku, U. Pinsopon, S. Cetinkunt, and S. Nakajima, "Design, fabrication, and real-time neural network control of a three-degreesof-freedom nanopositioner," IEEE/ASME Transactions on Mechatronics, vol. 5, no. 3, pp. 273-280, September 2000.

[2] K. Spanner and S. Vorndran, "Advances in piezo-nanopositioning technology," in Proc. IEEE/ASME International Conference on Advanced Intelligent Mechatronics, vol. 2, Kobe, Japan, 20-24 July 2003, pp. 1338-1343.

[3] B. J. Yi, G. B. Chung, H. Y. Na, W. K. Kim, and I. H. Suh;, "Design and experiment of a 3-dof parallel micromechanism utilizing flexure hinges," IEEE Transactions on Robotics and Automation, vol. 19, no. 4, pp. 604-612, August 2003.

[4] Y. I. Somov, "Modelling physical hysteresis and control of a fine piezodrive," in Proc. International Conference Physics and Control, vol. 4, St. Petersburg, Russia, 20-22 August 2003, pp. 1189-1194.

[5] T. S. Low and W. Guo, "Modeling of a three-layer piezoelectric bimorph beam with hysteresis," Journal of Microelectromechanical Systems, vol. 4, no. 4, pp. 230-237, December 1995.

[6] M. Goldfarb and N. Celanovic, "Modeling piezoelectric stack actuators for control of micromanipulation," IEEE Control Systems Magazine, vol. 17, no. 3, pp. 69-79, June 1997.

[7] R. Banning, W. L. D. Koning, H. J. M. T. A. Adriaens, and R. K. Koops, "State-space analysis and identification for a class of hysteretic systems," Automatica, vol. 37, no. 12, pp. 1883-1892, December 2001.

[8] H. J. M. T. A. Adriaens, W. L. D. Koning, and R. Banning, "Modeling piezoelectric actuators," IEEE/ASME Transactions on Mechatronics, vol. 5, no. 4, pp. 331-341, December 2000.

[9] T. Chang and X. Sun, "Analysis and control of monolithic piezoelectric nano-actuator," IEEE Transactions on Control Systems Technology, vol. 9, no. 1, pp. 69-75, January 2001.

[10] H. J. Shieh, F. J. Lin, P. K. Huang, and L. T. Teng, "Adaptive tracking control solely using displacement feedback for a piezo-positioning mechanism," IEE Proc. Control Theory and Applications, vol. 151, no. 5, pp. 653-660, September 2004.

[11] Y. C. Huang and C. H. Cheng, "Robust tracking control of a novel piezodriven monolithic flexure-hinge stage," in Proc. IEEE International Conference on Control Applications, vol. 2, Taipei, Taiwan, 2-4 September 2004, pp. 977-982.

[12] G. Schitter and A. Stemmer, "Identification and open-loop tracking control of a piezoelectric tube scanner for high-speed scanning-probe microscopy," IEEE Transactions on Control Systems Technology, vol. 12, no. 3, pp. 449-454, May 2004.

[13] C. L. Hwang, Y. M. Chen, and C. Jan, "Trajectory tracking of largedisplacement piezoelectric actuators using a nonlinear observer-based variable structure control," IEEE Transactions on Control Systems Technology, vol. 13, no. 1, pp. 56-66, January 2005.

[14] S. Yu, B. Shirinzadeh, G. Alici, and J. Smith, "Sliding mode control of a piezoelectric actuator with neural network compensating rate-dependent hysteresis," in Proc. IEEE International Conference on Robotics and Automation, Barcelona, Spain, 18-22 April 2005, pp. 3652-3656.

[15] J. Y. Hung, W. B. Gao, and J. C. Hung, "Variable structure control: a survey," IEEE Transactions on Industrial Electronics, vol. 40, no. 1, pp. 2-22, 1993.

[16] J. J. E. Slotine and W. Li, Applied Nonlinear Control. Englewood Cliffs, NJ: Prentice-Hall, 1991.

[17] J. J. Craig, Introduction to Robotics: Mechanics and Control. Reading, MA: Addison-Wesley, 1989.

[18] S. Vorndran, Ed., MicroPositioning, NanoPositioning, NanoAutomation: Solutions for Cutting-Edge Technologies. Karlsruhe, Germany: The PI-Polytec Group, 2001. 